\title{
Entanglement Renormalization for Quantum Fields in Real Space
}

\author{
Jutho Haegeman, ${ }^{1}$ Tobias J. Osborne, ${ }^{2}$ Henri Verschelde, ${ }^{1}$ and Frank Verstraete ${ }^{3,4}$ \\ ${ }^{1}$ Ghent University, Department of Physics and Astronomy, Krijgslaan 281-S9, B-9000 Ghent, Belgium \\ ${ }^{2}$ Institut für Theoretische Physik, Leibniz. Universität Hannover, Appelstrasse 2, 30167 Hannover, Germany \\ ${ }^{3}$ University of Vienna, Faculty of Physics, Boltzmanngasse 5, A-1090 Wien, Austria \\ ${ }^{4}$ C.N. Yang Institute for Theoretical Physics, SUNY, Stony Brook, New York 11794-3840, USA \\ (Received 16 June 2011; revised manuscript received 4 February 2013; published 5 March 2013)
}

\begin{abstract}
We show how to construct renormalization group (RG) flows of quantum field theories in real space, as opposed to the usual Wilsonian approach in momentum space. This is achieved by generalizing the multiscale entanglement renormalization ansatz to continuum theories. The variational class of wave functions arising from this RG flow are translation invariant and exhibits an entropy-area law. We illustrate the construction for a free nonrelativistic boson model, and argue that the full power of the construction should emerge in the case of interacting theories.
\end{abstract}

DOI: 10.1103/PhysRevLett.110.100402

Classical statistical mechanics, quantum many-body systems, and relativistic quantum field theories all involve an extremely large number of degrees of freedom living at different length scales. The interactions between these degrees of freedom are the source of notorious difficulties in their study. However, much insight has been gained from the renormalization group (RG), which has proven to be the natural tool to deal with the different length scales in such systems [1]. In its original development, the RG acts as a fixed operation at the level of the classical partition function (or related quantities such as the effective action). This operation is typically formulated in momentum space and is usually only exactly implementable for free theories. Perturbative expansions in a small parameter are required for interacting theories. In addition, this formulation of the RG is only applicable to quantum systems using the quantum-to-classical mapping, which is known to fail in some cases [2]. (See, e.g., Ref. [3], however, for important nonperturbative approaches overcoming these limitations.) Importantly, in its original incarnation, the RG is not a variational method.

One exception is Wilson's numerical renormalization group [1], which can be interpreted as an implementation of the RG directly at the level of the quantum wave function. Together with White's more powerful density matrix renormalization group [4], these methods are now understood as a variational optimization over the class of matrix product states [5]. Based on the observation of an entropy-area law [6] in locally interacting quantum lattices, quantum-information-theoretical considerations have resulted in the development of other sophisticated variational classes for quantum lattice systems. These are generally known as tensor network states and can be associated with RG schemes, allowing the classification of gapped phases of matter [7]. Unlike Wilson's fixed RG scheme, these are variable RG schemes that can be variationally optimized. They are formulated in real-space and
PACS numbers: 03.65.Ud, 03.67.Mn, 11.10.Kk, 11.25.Tq

deal equally well with free and interacting systems. One specific scheme, called entanglement renormalization [8], can also be applied to study critical phases and can be used to compute, e.g., scaling exponents [9]. The corresponding variational class, the multiscale entanglement renormalization ansatz (MERA) [10], is set apart by its unique properties, including, the ability to support algebraically decaying correlations and logarithmic corrections to the entropy-area law in $1+1$ dimensions. This class has been successfully applied to study the physics of a wide variety of strongly interacting systems in low dimensions, including the study of real-time evolution, and fermionic and anyonic systems which are inaccessible by Monte Carlo methods [10-13].

Most of these developments have been restricted to the lattice setting. While they do allow the study of continuous quantum systems via discretization, it is often desirable to work directly in the continuum. Examples in condensed matter physics include strongly interacting ultracold atomic gases [14] and impurity problems [1,15], whereas the fermion doubling problem [16] clearly motivates a continuum treatment of relativistic theories. Recently it was understood how to define matrix product states and its higherdimensional analog for continuous systems [17]. This class has already been successfully applied to study both nonrelativistic and relativistic quantum field theories [18]. It is the objective of this Letter to define a generalization of MERA directly in the continuum. The area law for entanglement entropy-with logarithmic violations for critical theories in $1+1$ dimensions-has also been observed in the continuum in the context of black hole physics [19] and conformal field theories [20], and thus validates the potential usefulness of our approach. Additionally, the entanglement content of our continuum ansatz confirms holographic proposals for entanglement entropy [21].

The MERA class.-The MERA construction, introduced in Ref. [10], may be described either via an active renormalization process applied to a strongly correlated quantum 
state or, dually, as the result of a special quantum circuit applied to a simple fiducial state. This reverse description is not in violation with the irreversibility of the RG, since it only applies to the ground state, not to the whole Hilbert space and the Hamiltonian acting upon it. At stage 0 of the MERA construction, the degrees of freedom (distinguishable quantum spins in this case) are arranged in a regular lattice and initialized in some convenient initial state, e.g., the "all 0s" state $|\mathbf{0}\rangle$. At stage 1 the degrees of freedom are subjected to a local interaction $U_{1}$ for some constant time, resulting in a correlated quantum state $U_{1}|\mathbf{0}\rangle$ of the lattice. The precise details of $U_{1}$-while playing a key role in applications of the variational principle - are not required to establish the general properties of a MERA. At the next stage the lattice is subjected to a scale transformation and the lattice spacing is doubled. At this point, in order to renormalize the lattice (i.e., restore the lattice spacing), new degrees of freedom are introduced: one adds a quantum spin initialized in the state $|0\rangle$ between each pair of the old lattice sites. We write this renormalization step as $\mathcal{R}$. The resulting state $\mathcal{R} U_{1}|\mathbf{0}\rangle$ is then again subjected to a local interaction $U_{2}$ for some constant time followed by the renormalization step $\mathcal{R}$, producing the state $\mathcal{R} U_{2} \mathcal{R} U_{1}|\mathbf{0}\rangle$. This process is then iterated as many times as desired or required. A physical interpretation of the MERA construction is straightforward. Every layer $k$ begins with a dilation of the state living in the previous layer to a lattice doubled in size, by applying $\mathcal{R}$. The operation of $U_{k}$ then adds short-range (e.g., over two sites) fluctuations or entanglement on top of this state, corresponding to fluctuations over $2^{m-k}$ sites in the final state if $m$ layers are applied. However, the resulting state $\left|\Psi_{\text {MERA }}\right\rangle=$ $U_{m} \mathcal{R} U_{m-1} \mathcal{R} \ldots \mathcal{R} U_{1}|\mathbf{0}\rangle$ generally breaks translation invariance unless the $U_{j}$ are carefully fine tuned.

The passage to the continuum.-For simplicity we specialize to the case of a single bosonic species in one dimension. (The generalization to higher dimensions and to fermions or Bose-Fermi mixtures is entirely straightforward.) We write $\psi(x)$ and $\psi^{\dagger}(x), x \in \mathbb{R}$, for the bare field annihilation and creation operators which obey the canonical commutation relations $\left[\psi(x), \psi^{\dagger}(y)\right]=\delta(x-y)$. The following constructions can also be described in terms of any set of operators that define the theory, such as the Hermitian field operator $\phi$ and its conjugate momentum $\pi$ for relativistic boson theories.

The generalization of the 0th stage of the MERA construction is clear: one should choose for the initial state a factorized reference state $|\Omega\rangle$. The transition to a continuous space $x \in \mathbb{R}$ enables the introduction of a continuous scale parameter $s$ that labels the layers of the MERA construction. In every layer, new fluctuations are created by the action of a unitary evolution $U(s)=\exp (-i \delta K(s))$ with time step $\delta$ and local interaction $K(s)$ given by

$$
K(s)=\int k(x, s) d x
$$

where $k(x, s)$ is a local combination of the field operators $\psi(x)$, their adjoints, and their derivatives. Since the continuum lacks a shortest distance, we need to introduce a characteristic length scale $\boldsymbol{\epsilon}$ below which $K(s)$ does not create entanglement. This is possible in a variety of ways: one strategy is to build $k(x)$ from smoothed field operators $\tilde{\psi}_{\epsilon}(x)=\int \chi_{\epsilon}(x-y) \psi(y) d y$, where $\chi_{\epsilon}(x)$ is some smooth envelope function which is nonzero outside a region of width $\epsilon$ around $x=0$. Another strategy is to simply impose a cutoff on $K$ when it is expressed in terms of momentum variables. The cutoff $\Lambda \approx \epsilon^{-1}$ ensures that the only degrees of freedom which are nontrivially affected are those with momenta $k \lesssim \Lambda$.

The third ingredient of the MERA construction is the renormalization step where the scale is changed and new uncorrelated degrees of freedom are introduced. Here the continuum analog is not entirely clear, but we argue that the following replacement naturally achieves the same objective: we simply effect a small change of scale via $W=\exp (-i \delta L)$, where the generator $L$ is given by

$$
L=-\frac{i}{2} \int \psi^{\dagger}(x) x \frac{d \psi(x)}{d x}-x \frac{d \psi^{\dagger}(x)}{d x} \psi(x) d x .
$$

This has the same physical outcome as the original MERA renormalization step because initially uncorrelated degrees of freedom at length scales below $\epsilon$ are now introduced at the new length scale. Thus, our proposal for the continuous MERA (cMERA) is as follows: evolve some initial state $|\Omega\rangle$ according to $K$ for an infinitesimal time $\delta$, which correlates real-space degrees of freedom at scales of $\mathcal{O}(\epsilon)$. Then introduce new degrees of freedom from the higher momenta or shorter length scales by dilating the state via evolution according to $L$ for a time $\delta$. The last layer $s=s_{\epsilon}$ creates fluctuations at length scale $\epsilon$. No fluctuations at a shorter range exist in the final state. The fluctuations created by layer $s$ live at length scale $\epsilon \exp \left(s_{\epsilon}-s\right)$ in the final state. If this process is to correctly produce the long-range entanglement in a state with correlation length $\xi$ the first layer should be defined at $s=s_{\xi}$ with $s_{\epsilon}-s_{\xi}=\mathcal{O}(\log (\xi / \epsilon))$. By taking the limit $\delta \rightarrow 0$ we obtain our final expression

$$
|\Psi\rangle \equiv \mathcal{T} e^{-i \int_{s_{\xi}}^{s_{\epsilon}} K(s)+L d s}|\Omega\rangle \equiv U\left(s_{\epsilon}, s_{\xi}\right)|\Omega\rangle,
$$

where $\mathcal{T}$ is the time-ordering operation. We refer to the unitary operation preparing a cMERA as $U\left(s_{\epsilon}, s_{\xi}\right)$. Note that a UV cutoff $\Lambda=\epsilon^{-1}$ and an IR cutoff $\xi^{-1}$ are explicitly built into the cMERA definition. For critical systems or relativistic theories with an infinite range of fluctuations it is necessary for $s_{\epsilon}-s_{\xi} \rightarrow \infty$.

The biggest difference between the cMERA and MERA definitions arises from the flexibility we have in imposing the UV cutoff. In the MERA case the UV cutoff is dictated by the lattice spacing. This, in turn, essentially forces the form of the subsequent scaling transformation (i.e., an 
integral number of spins must be introduced in the scaling step). The freedom, arising from the continuum, to choose a smooth UV cutoff allows the scaling transformation to be applied continuously and for the resulting state to be easily chosen to be translation invariant.

The set of all cMERA forms a variational class: the variational parameters are the coefficients of interactions involved in $K$; these coefficients may depend on both $x$ and the integration parameter $s$. When the coefficients do not depend on position $x$ a generic cMERA is manifestly translation invariant (this is established by noting that application of the unitary $e^{i \delta L}$ to a translation-invariant state results in a translation invariant state). This is in contradistinction to the generic situation with lattice MERA.

Comparison with Wilsonian renormalization.-The cMERA has been constructed using the quantum circuit interpretation, but can now be interpreted as an active renormalization process and compared to Wilson's momentum-shell renormalization group (RG) [1]. The latter works by iteratively integrating out all the modes living in a small momentum shell $\Lambda-d \Lambda<|\vec{k}|<\Lambda$. A rescaling step brings modes at $\Lambda-d \Lambda$ back to $\Lambda$. In the end $\Lambda$ can be sent to infinity, but we need to start with a finite $\Lambda$ in order to define the process. The renormalization process defined by the cMERA proposal is a variational real-space implementation of Wilsonian renormalization in a Hamiltonian framework: rather than integrating out highfrequency modes around the cutoff $\Lambda$ from the partition function - which is a fixed operation - the operator $K$ first disentangles these modes from the wave function in such a way that they can be isometrically projected onto a reference vacuum $|\Omega\rangle$. Then a scale transformation is performed to send the disentangled modes beyond the cutoff (where they no longer interact via $K$ ) and new entangled modes are brought to $\Lambda$. These modes are then disentangled in the subsequent step. This immediately clarifies the need for a finite cutoff $\Lambda$ in our construction (which can also be sent to infinity at the end of the process).

So what are the differences between the renormalization process defined by the cMERA and Wilson's momentumshell RG? First, whereas Wilson's RG is a fixed operation, the cMERA renormalization process is governed by $K(s)$ which can be variationally optimized. Second, while $K(s)$ can be formulated in momentum space (e.g., to implement the cutoff), its defining property is real-space locality, which has proven to be a correct assumption for both free and interacting theories in countless examples with MERA and related variational classes and is a result of the locality of physical interactions.

The cMERA RG flow.-New to our formalism is that we can define the RG flow for operators in a Hamiltonian framework: suppose we want to evaluate the expectation value $\langle O\rangle=\langle\Psi|O| \Psi\rangle$, where $|\Psi\rangle$ is a cMERA of the form Eq. (3), and $O$ is some local operator, e.g., $O=\psi(0)$ or
$O=\psi^{\dagger}(0) \psi(x)$. To do this we define $O(s) \equiv$ $U\left(s_{\epsilon}, s\right)^{\dagger} O U\left(s_{\epsilon}, s\right)$, where

$$
\frac{d O(s)}{d s}=-i[K(s)+L, O(s)] .
$$

This "Heisenberg-like" equation of motion is obtained by differentiating the lower limit of the evolution operator $U\left(s_{\epsilon}, s\right)$. Then $\langle O\rangle$ may be found by integrating this equation from $s=s_{\epsilon}$ down to $s=s_{\xi}$, with the initial condition $O\left(s_{\epsilon}\right)=O$, and evaluating $\left\langle\Omega\left|O\left(s_{\xi}\right)\right| \Omega\right\rangle$.

Physically, we think of the bare or physical operator $O$ as being defined at the UV cutoff length scale $x \sim \epsilon$. As "time" $s$ progresses we think of $O(s)$ as being brought from length scale $x \sim \epsilon$ to length scale $x \sim \epsilon e^{s_{\epsilon}-s}$. Thus, $O(s)$ is obtained from the bare operator $O$ by renormalizing up to scale $s$ (i.e., all degrees of freedom between momentum scales $e^{s-s_{\epsilon}} \Lambda$ and $\Lambda$, where $s<s_{\epsilon}$ have been integrated out or disentangled).

For critical theories, $K(s)$ is expected to become $s$ independent away from $s_{\epsilon}$. In accordance with the results from Ref. [9], we can then assume the existence of operators $O$ that satisfy $-i[K+L, O]=\lambda O$. These are scaling operators with scaling dimension $\lambda$. If $O$ is a local scaling operator, it is necessarily centered around $x=0$. A local scaling operator $O(x)$ with scaling dimension $\lambda$ at position $x$ satisfies

$$
-i[K+L, O]=x d O(x) / d x+\lambda O(x),
$$

where the commutator with $L$ generates the canonical scaling dimension and the contribution of $K$ can be understood as generating the anomalous scaling dimension. The existence of scaling operators makes it easy to prove that cMERA support algebraically decaying correlations and are thus well suited to study critical theories. In addition, we can illustrate that they are able to produce an area law for the entanglement entropy.

An entropy-area law for cMERA.-We now provide a heuristic argument that a generic cMERA obeys an entropy-area law by appealing to results [22] concerning the dynamics of quantum spin systems: it is known that the entanglement entropy $S_{A}(t)=-\operatorname{tr}\left(\rho_{A}(t) \log \left(\rho_{A}(t)\right)\right.$, where $\rho_{A}(t)=\operatorname{tr}_{A^{c}}\left(e^{-i t H}\left|\phi_{0}\right\rangle\left\langle\phi_{0}\right| e^{i t H}\right)$ is the reduced density operator for a region $A$, under the real-time dynamics generated by any strongly interacting system grows as

$$
d S_{A}(t) / d t \leq c|\partial A|,
$$

where $|\partial A|$ denotes the length or area of the boundary of $A$ and should be measured in terms of the cutoff of $H$, for some constant $c$ which depends only on the local interactions and the geometry of $A$. It is natural to conjecture that this result holds in the continuum setting. Now, subject to this assumption, we can deduce the proposed area law: we track the entropy growth of the time-dependent region $A(s)=A e^{s-s_{\epsilon}}$, i.e., $A(s)$ is $A$ scaled down by a factor $e^{s-s_{\epsilon}}$ throughout the cMERA preparation. We bound the 
entropy $S_{A}$ by integrating Eq. (6) (compare with $[21,23,24])$ :

$$
\begin{aligned}
S_{A} & \leq c \int_{s_{\epsilon}-\log (L \Lambda)}^{s_{\epsilon}}\left(L \Lambda e^{s-s_{\epsilon}}\right)^{d-1} d s \\
& = \begin{cases}c \log (L \Lambda), & d=1 \\
\frac{c}{d-1}(L \Lambda)^{d-1}\left(1-\frac{1}{(L \Lambda)^{d-1}}\right), & d>1,\end{cases}
\end{aligned}
$$

where the area $|\partial A| \leq(L \Lambda)^{d-1}$; the entanglement of $A(s)$ with the remainder of the system cannot receive further contributions when $|A(s)| \leq(L \Lambda)^{d}<1$. The appearance of the logarithm of the UV cutoff is familiar from standard QFT calculations $[19,20]$. Note that the cMERA might not describe logarithmic violations of the boundary law in $d>1$, similar to the MERA case.

Representation of ground states via cMERA.-Let us now construct a cMERA representation of the ground state of a simple nonrelativistic bosonic model

$$
H=\int\left[\frac{d \psi^{\dagger}}{d x} \frac{d \psi}{d x}+\mu \psi^{\dagger} \psi-\nu\left(\psi^{\dagger 2}+\psi^{2}\right)\right] d x .
$$

which is well defined if $2 \nu \leq \mu$. For $2 \nu=\mu$, the elementary excitation becomes massless and the model becomes critical. Using a general strategy discussed in Ref. [25], we can describe ground states of free theories with a Gaussian cMERA, where $K(s)$ is the quadratic operator

$K(s)=-\frac{i}{2} \int g\left(\frac{k}{\Lambda}, s\right)\left[\hat{\psi}^{\dagger}(k) \hat{\psi}^{\dagger}(-k)-\hat{\psi}(-k) \hat{\psi}(k)\right] d k$,

where $\hat{\psi}(k)$ is the Fourier transform of the field operators. We set $g(k / \Lambda, s)=\chi(s) \Gamma(|k| / \Lambda)$ with $\Gamma(\kappa)$ a fixed cutoff function with cutoff 1 . The variational parameters are thus the function values $\chi(s) .|\Omega\rangle$ is fixed by $\psi|\Omega\rangle=0$. The analytic calculations are facilitated using a sharp cutoff such as $\Gamma(\kappa)=\theta(1-|\kappa|)$, where $\theta(x)$ is the Heaviside function. Although this cutoff function produces a nonlocal operator $K$, it is straightforward to see that similar results are obtained using a smooth cutoff such as $\Gamma(\kappa)=$ $\exp \left(-\kappa^{2}\right)$ which does yield a local $K$. The exact ground state of $H$ can be accurately reproduced [26] if $\Lambda^{2} \gg \mathcal{O}(\nu)$ by choosing

$$
\chi(s)=2\left(\nu / \Lambda^{2}\right) /\left[\left(e^{2 s}+\Delta^{2} / \Lambda^{4} e^{-2 s}\right)+2 \mu / \Lambda^{2}\right],
$$

where we have set $s_{\epsilon}=0$ and $\Delta=\left(\mu^{2}-4 \nu^{2}\right)^{1 / 2}$ represents the mass gap of the system. For $2 \nu<\mu$ or thus $\Delta>0$, the 'disentangling strength' $\chi(s)$ peaks at $s=$ $-1 / 2 \log \left(\Lambda^{2} / \Delta\right)$ and decays to zero for $s \rightarrow-\infty$. The integration of the RG flow can be stopped at $s_{\xi} \ll$ $-1 / 2 \log \left(\Lambda^{2} / \Delta\right)-\mathcal{O}\left(\mu / \Lambda^{2}\right)$. Figure 1 depicts the momentum occupation $n(p)$-obtained as Fourier transform of the spectral function $\left\langle\psi^{\dagger}(x) \psi(y)\right\rangle$-for different values of the integration limit $s_{\xi}$. In the critical limit $(2 \nu \rightarrow \mu), \chi(s)$ reaches a nonzero horizontal asymptote

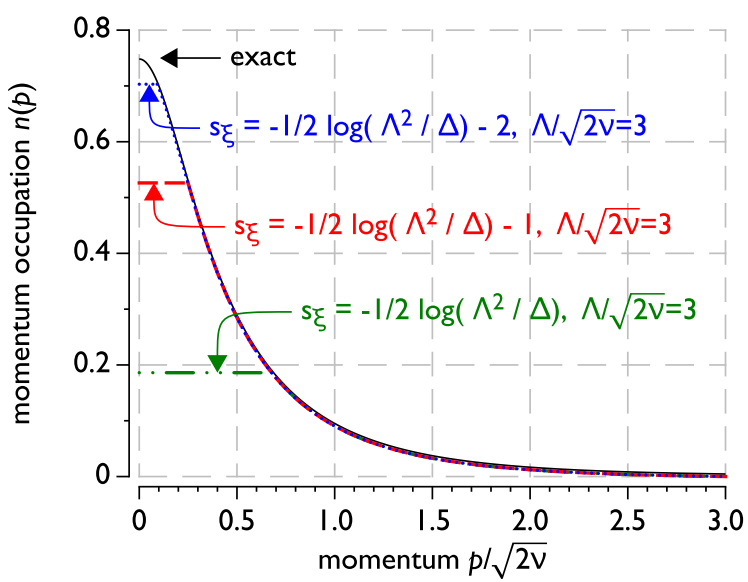

FIG. 1 (color online). The momentum occupation $n(p)$ for the cMERA approximation of the ground state of the Hamiltonian in Eq. (8) with $\mu / 2 \nu=1.1(\Delta / 2 \nu \approx 0.46)$ for different values of the integration limit $s_{\xi}$. Note that the error becomes negligible if $s_{\xi}$ is sufficiently larger than $-1 / 2 \log \left(\Lambda^{2} / \Delta\right)$ and that the UV error (vertical offset between exact and approximate results at large momentum) is completely invisible even for the moderate choice of the UV cutoff $\Lambda=3 \sqrt{2 \nu}$.

$\lim _{s \rightarrow-\infty} \chi(s)=\nu / \mu=1 / 2$ and we need to integrate all the way down to $s_{\xi}=-\infty$. According to Eq. (5), the scaling operators then correspond to $\phi(x) \sim(\psi(x)+$ $\left.\psi^{\dagger}(x)\right)$ and $\pi(x) \sim\left(\psi(x)-\psi^{\dagger}(x)\right)$. The low-energy behavior is scale invariant and can be described by the massless Klein-Gordon equation [25].

This example is a free Gaussian theory; however, interacting theories can also be variationally treated within our framework. Here one adds interacting terms to $K$, which are additional variational parameters, and computes expectation values by solving Eq. (4) variationally using an expansion of $O(s)$ in a power series of local operators and truncating it optimally. Divergences in the interacting theory must be dealt with as usual by only computing operationally defined cutoff independent quantities.

Conclusions.-In this Letter a generalization, cMERA, of the MERA variational class to the continuum setting has been introduced. We have argued that cMERA can be translation invariant and generically exhibit an entropyarea law. We have also supplied an analytic argument that the ground states of a general class of local quadratic models admits a cMERA description. Much remains to be done: we expect, by analogy with MERA, that cMERA will be a useful variational class for strongly interacting quantum fields, and will allow the description of a variety of interesting physical phenomena from topological effects to confinement, and symmetry breaking. Looking further afield, the cMERA constitutes a realization of the holographic principle. It is tempting to speculate, building on $[24,27]$, that cMERA is a natural candidate to establish a link between entanglement renormalization and the best known realization of the holographic principle, namely the AdS/CFT correspondence. 
This work was supported by the Research Foundation Flanders (J.H.), ERC grants QUERG, COQUIT, and QFTCMPS and the cluster of excellence EXC 201 Quantum Engineering and Space-Time Research. J.H. is grateful to T.J.O. for the invitation to the Leibniz Universität Hannover.

[1] K. G. Wilson, Rev. Mod. Phys. 47, 773 (1975).

[2] T. Senthil, A. Vishwanath, L. Balents, S. Sachdev, and M.P. A. Fischer, Science 303, 1490 (2004); M. Vojta, N.-H. Tong, and R. Bulla, Phys. Rev. Lett. 94, 070604 (2005).

[3] M. Asorey, J. G. Esteve, and J. Salas, Phys. Rev. B 48, 3626 (1993); F. J. Wegner, Ann. Phys. (N.Y.) 3, 77 (1994); S. D. Glazek and K. G. Wilson, Phys. Rev. D 48, 5863 (1993); S. Kehrein, Phys. Rev. Lett. 83, 4914 (1999); S. D. Glazek and K. G. Wilson, Phys. Rev. Lett. 89, 230401 (2002).

[4] S. R. White, Phys. Rev. Lett. 69, 2863 (1992); U. Schollwöck, Rev. Mod. Phys. 77, 259 (2005).

[5] M. Fannes, B. Nachtergaele, and R. F. Werner, Commun. Math. Phys. 144, 443 (1992); S. Östlund and S. Rommer, Phys. Rev. Lett. 75, 3537 (1995); F. Verstraete, J. I. Cirac, and V. Murg, Adv. Phys. 57, 143 (2008); J. I. Cirac and F. Verstraete, J. Phys. A 42, 504004 (2009).

[6] F. Verstraete and J.I. Cirac, Phys. Rev. B 73, 094423 (2006); T. J. Osborne, Phys. Rev. Lett. 97, 157202 (2006); M. B. Hastings, J. Stat. Mech. (2007) P08024; J. Eisert, M. Cramer, and M. B. Plenio, Rev. Mod. Phys. 82, 277 (2010).

[7] Z.-C. Gu, M. Levin, and X.-G. Wen, Phys. Rev. B 78, 205116 (2008); H. H. Zhao, Z. Y. Xie, Q. N. Chen, Z. C. Wei, J. W. Cai, and T. Xiang, Phys. Rev. B 81, 174411 (2010).

[8] G. Vidal, Phys. Rev. Lett. 99, 220405 (2007).

[9] V. Giovannetti, S. Montangero, and R. Fazio, Phys. Rev. Lett. 101, 180503 (2008); R. N. C. Pfeifer, G. Evenbly, and G. Vidal, Phys. Rev. A 79, 040301 (2009).

[10] G. Vidal, Phys. Rev. Lett. 101, 110501 (2008).

[11] G. Evenbly and G. Vidal, Phys. Rev. B 79, 144108 (2009); V. Giovannetti, S. Montangero, M. Rizzi, and R. Fazio,
Phys. Rev. A 79, 052314 (2009); G. Vidal, arXiv:0912.1651.

[12] L. Cincio, J. Dziarmaga, and M. M. Rams, Phys. Rev. Lett. 100, 240603 (2008); G. Evenbly and G. Vidal, Phys. Rev. Lett. 102, 180406 (2009); G. Evenbly and G. Vidal, Phys. Rev. Lett. 104, 187203 (2010).

[13] P. Corboz, G. Evenbly, F. Verstraete, and G. Vidal, Phys. Rev. A 81, 010303(R) (2010); C. Pineda, T. Barthel, and J. Eisert, Phys. Rev. A 81, 050303 (2010).

[14] I. Bloch, J. Dalibard, and W. Zwerger, Rev. Mod. Phys. 80, 885 (2008).

[15] P. W. Anderson, Phys. Rev. 124, 41 (1961).

[16] H. B. Nielsen and M. Ninomiya, Nucl. Phys. B185, 20 (1981); H. B. Nielsen and M. Ninomiya, Phys. Lett. 105B, 219 (1981).

[17] F. Verstraete and J. I. Cirac, Phys. Rev. Lett. 104, 190405 (2010); T. J. Osborne, J. Eisert, and F. Verstraete, Phys. Rev. Lett. 105, 260401 (2010).

[18] J. Haegeman, J. I. Cirac, T. J. Osborne, H. Verschelde, and F. Verstraete, Phys. Rev. Lett. 105, 251601 (2010); Proc. Sci. (2010), 029.

[19] L. Bombelli, R. K. Koul, J. Lee, and R. D. Sorkin, Phys. Rev. D 34, 373 (1986); M. Srednicki, Phys. Rev. Lett. 71, 666 (1993).

[20] C. G. Callan and F. Wilczek, Phys. Lett. B 333, 55 (1994); C. Holzhey, F. Larsen, and F. Wilczek, Nucl. Phys. B300, 377 (1988); P. Calabrese and J. Cardy, J. Stat. Mech. (2004) P06002.

[21] S. Ryu and T. Takayanagi, Phys. Rev. Lett. 96, 181602 (2006); J. High Energy Phys. 08 (2006) 045.

[22] S. Bravyi, M. B. Hastings, and F. Verstraete, Phys. Rev. Lett. 97, 050401 (2006); J. Eisert and T. J. Osborne, Phys. Rev. Lett. 97, 150404 (2006).

[23] G. Vidal, Phys. Rev. Lett. 101, 110501 (2008).

[24] B. Swingle, Phys. Rev. D 86, 065007 (2012).

[25] J. Haegeman, T. J. Osborne, H. Verschelde, and F. Verstraete (to be published).

[26] The fidelity $F=\left|\left\langle\Psi_{\text {exact }} \mid \Psi_{\text {cMERA }}\right\rangle\right|$ is given by $F=$ $\exp [-(L \Lambda) f]$ with $L \rightarrow \infty$ the diverging system size and the local fidelity density $f \sim 2 \nu / \Lambda^{2}$.

[27] G. Evenbly and G. Vidal, J. Stat. Phys. 145, 891 (2011). 